\title{
Future of Biotransformation Science in the Pharmaceutical Industry
}

Valerie M. Kramlinger ${ }^{1}$, Deepak Dalvie ${ }^{2, \#}$, Carley J.S. Heck ${ }^{3}$, Amit S. Kalgutkar ${ }^{4}$, James

O’Neill ${ }^{5}$, Dian Su ${ }^{6, \%}$, Aaron M. Teitelbaum ${ }^{7}$, and Rheem A. Totah ${ }^{8}$

All authors contributed equally.

\author{
Affiliations: \\ ${ }^{1}$ Translational Medicine, Novartis Institutes for Biomedical Research, Inc., Cambridge, MA, \\ 02139, USA \\ ${ }^{2}$ Bristol Myers Squibb, San Diego CA 92121 \\ ${ }^{3}$ Medicine Design, Pfizer Worldwide Research, Development and Medical, Eastern Point Road, \\ Groton, CT 06340 \\ ${ }^{4}$ Medicine Design, Pfizer Worldwide Research, Development and Medical, 1 Portland St, \\ Cambridge, MA 02139 \\ ${ }^{5}$ Charles River Laboratories Edinburgh Ltd, Elphinstone Research Centre, Tranent, East Lothian \\ EH33 $2 \mathrm{NE}^{6}$ Department of Drug Metabolism and Pharmacokinetics, Genentech, Inc., 1 DNA \\ Way, South San Francisco, CA 94080; \\ ${ }^{7}$ Drug Metabolism and Pharmacokinetics, Boehringer Ingelheim Pharmaceuticals, Inc., \\ Ridgefield, CT, 06877 \\ ${ }^{8}$ Medicinal Chemistry, University of Washington \\ Running Title: Industry opportunities for Biotransformation Scientists
}


DMD-MR-2021-000658

Corresponding Author: Valerie Kramlinger; valerie.kramlinger@novartis.com; 1-612-598-

4018. Translational Medicine, Novartis Institutes for Biomedical Research, Inc., Cambridge, MA, 02139, USA

\section{Orchid ID}

Valerie M. Kramlinger: https://orcid.org/0000-0002-7237-164X

Deepak Dalvie: Not applicable

Carley J.S. Heck: https://orcid.org/0000-0002-6842-3670

Amit Kalgutkar: https://orcid.org/0000-0001-9701-756X

James O’Neill: Not applicable

Dian Su: https://orcid.org/0000-0003-4572-462X

Aaron M. Teitelbaum: https://orcid.org/0000-0001-6737-0928

Rheem A. Totah: https://orcid.org/0000-0003-1664-4874

Number of text pages: 24

Number of words in the abstract: 239

Number of words in the introduction: 423

Number of words in the discussion: 6340

Number of references: 46

Number of tables: 0

Number of figures: 6 
DMD-MR-2021-000658

\section{Abbreviations}

ADC, antibody drug conjugate; ADH/ALDH, alcohol dehydrogenase/aldehyde dehydrogenase;

ADME, absorption, distribution, metabolism, and excretion; AKR, aldo-keto reductase; BMPFG, biotransformation, mechanisms, and pathways focus group; $\mathrm{CL}_{\mathrm{int}}$, intrinsic clearance; $\mathrm{CL}_{\mathrm{p}}$, plasma clearance; CBR, carbonyl reductase; CRO, contract research organization; CYP, cytochrome P450; DMPK, drug metabolism/pharmacokinetics; GSH, reduced glutathione; HLM, human liver microsomes, HPLC, high performance liquid chromatography; $\beta$-HSD, $\beta$ hydroxysteroid dehydrogenase; ISSX, international society for the study of xenobiotics; LCMS/MS, liquid chromatography tandem mass spectrometry; MAO, monoamine oxidase; MIST, metabolites in safety testing; NIH, national institutes of health 4-OH-CTA, 4hydroxycrotonaldehyde; PK/PD, pharmacokinetics/pharmacodynamics; PROTAC, proteolysis targeting chimera; SAR, structure-activity relationship; $\mathrm{t}_{1 / 2}$, half-life. 
DMD-MR-2021-000658

\begin{abstract}
Over the past decades, the number of scientists trained in departments dedicated to traditional medicinal chemistry, biotransformation and/or chemical toxicology have seemingly declined. Yet, there remains a strong demand for such specialized skills in the pharmaceutical industry, particularly within drug metabolism/pharmacokinetics (DMPK) departments. In this position paper, the members of the Biotransformation, Mechanisms, and Pathways Focus Group (BMPFG) steering committee reflect on the diverse roles and responsibilities of scientists trained in the biotransformation field in pharmaceutical companies and contract research organizations. The BMPFG is affiliated with the International Society for the Study of Xenobiotics (ISSX) and was specifically created to promote the exchange of ideas pertaining to topics of current and future interest involving the metabolism of xenobiotics (including drugs). The authors also delve into the relevant education and diverse training skills required to successfully nurture the future cohort of industry biotransformation scientists and guide them towards a rewarding career path. The ability of scientists with a background in biotransformation/organic chemistry to creatively solve complex drug metabolism problems encountered during research and development efforts on both small molecule or large molecular modalities is exemplified in five relevant case studies. Finally, the authors stress the importance and continued commitment to training the next generation of biotransformation scientists who are not only experienced in the metabolism of conventional small molecule therapeutics, but are also equipped to tackle emerging challenges associated with new drug discovery modalities including peptides, protein degraders and antibodies.
\end{abstract}




\section{DMD-MR-2021-000658}

Significance Statement: Biotransformation and mechanistic drug metabolism scientists are critical to advancing chemical entities through discovery and development, yet the number of scientists academically trained for this role is on the decline. This position paper highlights the continuing demand for biotransformation scientists and the necessity to nurture creative ways to train them and guarantee the future growth of this field. 
DMD-MR-2021-000658

\section{Introduction}

Graduate research activities involving mechanistic studies on xenobiotic toxicity invariably expose students to the field of drug metabolism, particularly in cases where xenobiotics are enzymatically bioactivated to electrophilic reactive metabolites that can covalently adduct to proteins and DNA and cause toxicity. Likewise, graduate students who receive their training in medicinal chemistry, not only learn the art of organic synthesis as applied to rational design and synthesis of enzyme substrates/inhibitors (or receptor agonists/antagonists) for structure-activity relationship (SAR) analyses, but also gain an appreciation for biochemical screening and drug metabolism through their graduate coursework and potentially hands-on experience in the laboratory. During their training, medicinal chemists are also taught to apply physical and mechanistic organic chemistry concepts (e.g., the concepts of valence electrons, resonance effects, acidity, nucleophilicity, electrophilicity, etc.) in rationalizing the biochemistry of enzyme-catalyzed reactions, and even understand the chemical basis for conversion of a substrate into product. To round off their research training, most medicinal chemistry and biotransformation/chemical toxicology students will acquire the skillset of handling analytical instrumentation (HPLC, mass spectrometry, and/or NMR spectroscopy) to support their research projects (e.g., purification and characterization of their synthetic target compounds, biochemical assays to study xenobiotic metabolism, etc.). A common entry point for these graduates in a pharmaceutical company is in a laboratory-based role within specialized biotransformation groups supporting metabolite identification needs for drug discovery and development. Such groups largely exist across medium-to-large size pharmaceutical companies and contract research organizations (CROs). Medium-to-large size pharmaceutical companies can be loosely defined as organizations involved in drug discovery and early to late-stage clinical development 
DMD-MR-2021-000658

and have significant clinical, manufacturing, marketing, and commercial activities with a sizable workforce. It is not uncommon for companies to compete to identify, and successfully hire, candidates who can perform biotransformation-focused studies in support of drug discovery and development, which highlights the need for increased academic preparation that emphasizes the constellation of skills and knowledge described above. In this position paper, the members of the Biotransformation, Mechanisms, and Pathways Focus Group (BMPFG) steering committee, affiliated with the International Society for the Study of Xenobiotics (ISSX), aim to 1) use key examples to highlight the importance of biotransformation scientists in advancing drug candidates to the clinic at every stage of drug development, including their role in the everchanging landscape of new drug modalities, 2) provide an overview on the different industry career choices available including the role of drug hunter, 3) emphasize the key skills desired to be successful and 4) summarize the challenges and opportunities to ensure the future growth and prosperity of biotransformation science.

\section{Key Examples of Biotransformation Support for Small Molecules, and New}

\section{Modalities, in Drug Discovery and Development}

\section{Drug discovery}

Biotransformation scientists play a crucial role in the optimization of the absorption, distribution, metabolism, and excretion (ADME) attributes at both the hit-to-lead and lead-to-candidate identification phases of preclinical drug discovery. Extensive metabolism of early chemical hits/leads, which is reflected by a high metabolic intrinsic clearance $\left(\mathrm{CL}_{\text {int }}\right)$ in substrate depletion assays utilizing liver microsomes and/or hepatocytes from animals and humans, is a frequent liability encountered in early drug discovery programs. In vivo, this trait usually manifests in the 
form of high plasma clearance $\left(\mathrm{CL}_{\mathrm{p}}\right)$, short elimination half-life $\left(\mathrm{t}_{1 / 2}\right)$, and poor oral bioavailability arising from extensive first pass metabolism of the chemical hits/leads along the gastrointestinal tract and liver by oxidative (e.g., CYP) and conjugative metabolism (e.g., uridine glucuronosyl transferases and sulfotransferases) (Lin and Lu, 1997). Medicinal chemistry tactics to modulate the lipophilicity and/or incorporate structural features that render metabolically labile compounds resistant to metabolism requires both intellectual and experimental assistance from biotransformation scientists and subject matter experts alike. In a drug discovery setting, biotransformation scientists work closely with medicinal chemistry designers and discovery DMPK representatives to gain a comprehensive understanding of the issues (e.g., high metabolic $\mathrm{CL}_{\text {int }}$ in intestinal or liver microsomes, disconnect in in vitro-in vivo-correlation for $\mathrm{CL}_{\mathrm{p}}$ in animals (Hosea et al., 2009), disconnect in $\mathrm{CL}_{\text {int }}$ between liver microsomes versus hepatocytes, (Di et al., 2012) etc.) plaguing the chemical series, and are able to propose and execute targeted metabolite identification studies to assess the underlying cause(s).

Oftentimes, biotransformation scientists are able to accurately predict the major sites of metabolism and even provide some insights into the metabolizing enzymes involved, even before the experiment is conducted. First, they tend to rationalize ADME data using chemical intuition, much to the liking of their medicinal chemistry colleagues. An example of this behavior is the prospective prediction of metabolic soft spots (and the drug metabolizing enzymes involved) through visual inspection of chemical structures, which is eventually backed with experimental data. In other cases, using their drug metabolism knowledge base, they are able to predict the potential for oxidative metabolism catalyzed by CYP enzymes versus non-CYP enzymes (e.g., MAO, aldehyde oxidase, flavin monooxygenases, etc.), and focus their efforts towards targeted studies. This is an important aspect in drug design because the data allows medicinal chemists to 
choose the right biochemical system (e.g., liver microsomes for CYPs and flavin monooxygenases, hepatocytes for aldehyde oxidase and MAO, etc.) for optimization of metabolic stability. Given their passion for chemistry and chemical structures, such individuals, when faced with ADME issues in their programs, are often times able to rationalize (and potentially propose a solution) via the efficient use of published information on analogous structures (or sub-structures). Finally, depending on their understanding of pharmacology SAR trends in a discovery program, they are also able to pinpoint potentially active metabolites based on metabolite identification studies on lead compounds.

Metabolite identification studies are designed with a specific issue, to be studied, in mind, and typically involve incubations of test compounds in cryopreserved hepatocytes, microsomal and S9 fraction from the small intestine, liver, lung, and/or kidney supplemented with the appropriate co-factors, plasma, blood, etc. Components from in vitro incubations are separated using LC-MS/MS, followed by the use of MS/MS fragmentation to gain insights into the structures of the metabolite(s) formed.

The biotransformation data guides medicinal chemists in the rational design of follow-up compounds, wherein strategic replacements (e.g., incorporation of metabolically stable substituents, introduction of polarity, increased rigidity, etc.) are introduced in lieu of the metabolically labile functional groups and the SAR for primary pharmacology and metabolic liability is reevaluated in an iterative fashion (Cerny et al., 2020; Shanu-Wilson et al., 2020). Optimization of Metabolic Stability: An example of such metabolism-guided drug discovery efforts is evident with the published account on $N$-arylsulfonamide-based $\gamma$-secretase inhibitors (Figure 1) (Stepan et al., 2011). Initial metabolite identification studies demonstrated that the principal cause for the high metabolic $\mathrm{CL}_{\text {int }}$ for the lead $\gamma$-secretase inhibitor 1 in NADPH- 
supplemented human liver microsomes (HLM) was due to extensive oxidations on the pendant cyclohexyl ring in a CYP3A4-dependent fashion. Reduction in molecular size and introduction of polarity were used as standard medicinal chemistry tactics to address the metabolic liability. Reduction in ring size (cyclohexyl $\mathbf{1} \rightarrow$ cyclobutyl 4) did not lead to a reduction in HLM CL int $_{\text {, }}$ and concomitantly generated metabolite identification data revealed that the cyclobutyl ring in $\mathbf{4}$ was the primary site of oxidation, in a manner similar to 1 . Replacement of the cycloalkyl ring with corresponding cyclic ether (tetrahydropyrans) variants (compounds $\mathbf{2}$ and $\mathbf{3}$ ) only nominally reduced $\mathrm{CL}_{\mathrm{int}}$ (compound 3 ) with oxidative metabolism occurring on the carbons $\alpha$ to the ether oxygen to afford hemiacetal derivatives as metabolites. Simultaneously decreasing ring size and inserting oxygen yielded oxetane derivatives (compounds 5 and 6) with significant reductions in

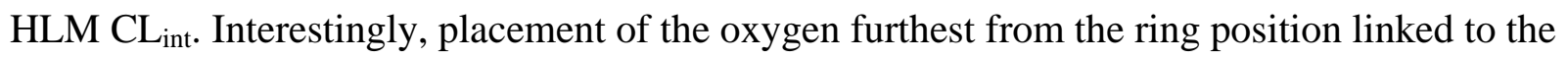
rest of the molecule yielded compound $\mathbf{6}$ with the lowest $\mathrm{CL}_{\text {int }}$ value in HLM. As such, all structural modifications to the cycloalkyl ring resulted in analogs that retained potency against the $\gamma$-secretase enzyme. The functional group changes introduced in the chemical series represent some empirically conceived insights regarding attenuating CYP-catalyzed metabolism by decreasing lipophilicity and reducing the size of cycloalkane rings, which coupled with metabolite identification studies offered a powerful approach in the near candidate quality design of $\gamma$-secretase inhibitors for the treatment of Alzheimer's disease.

Identification of Bio-reactive Metabolites: Apart from optimization of metabolic clearance and oral absorption, biotransformation studies are pivotal in assessing the metabolic activation (bioactivation) potential of hit/lead chemical entity to reactive metabolites, which can be a causative factor in certain toxicological outcomes associated with the parent precursors (Kalgutkar, 2020; Park et al., 2011). Because of the inability to predict and quantify the risk of 
toxicity arising from bioactivation, most pharmaceutical companies will trend towards elimination or considerable minimization of this liability in clinical candidates.

Biotransformation scientists assist in these activities by incubating test compounds in simple biochemical systems such as NADPH-supplemented HLM in the presence of a nucleophilic trapping agent (e.g., reduced glutathione $(\mathrm{GSH})$ ), followed by LC-MS/MS analysis of the incubation mixtures to detect the presence of adducts derived from the trapping of electrophilic metabolites by the included nucleophilic trap. Characterization of the adduct structure provides insights into the structure of the reactive species, and a mechanistic proposal for its formation. The information is then used by medicinal chemists to modify the structural features of the reactive metabolite positive compounds in order to eliminate the bioactivation liability (Kalgutkar, 2020). In practice, however, this activity is not trivial; medicinal chemistry solutions to eliminate bioactivation potential could result in an unfavorable effect on primary pharmacology and/or ADME attributes. Thus, tactics to eliminate the formation of reactive metabolites is an iterative process, the success of which is dependent on a close working relationship between biotransformation scientists and medicinal chemists.

An example where biotransformation studies were important in resolving bioactivation liabilities is evident with a series of electron-rich 5- aminooxindole-based inhibitors (e.g., compound 7, Figure 2) of the proline-rich tyrosine kinase 2 enzyme (Walker et al., 2008). The first step in this exercise was the visual recognition of the diaminophenyl structural alert, which is susceptible to a CYP-mediated two-electron oxidation to yield a reactive quinone-diimine species (Kalgutkar, 2020). Targeted studies to analyze the bioactivation potential of 7 via this pathway involved incubations in NADPH- and GSH-supplemented HLM, followed by LCMS/MS analysis of the incubation mixtures for metabolites (including reactive metabolites 
trapped by GSH). Mass spectral analysis revealed the formation of the GSH adduct $\mathbf{9}$, which is derived from addition of GSH to the electrophilic quinone-diimine intermediate 8 (Figure 2). The process of bioactivation with $\mathbf{7}$ was also accompanied by potent time- and NADPH-dependent inactivation of human CYP3A4 enzyme activity in HLM. In response to these observations, medicinal chemists introduced electron-deficient aniline ring substituents as five-membered lactam ring replacements, which led to compounds such as 10-13 that did not yield reactive species (inferred from the lack of formation of GSH adduct in metabolite identification studies), and did not cause CYP3A4 inactivation. In addition, analogs 10-13 demonstrated potent and selective inhibition of the kinase and metabolic stability in HLM, which was similar or greater than the corresponding 5-aminooxindole derivatives such as $\mathbf{7 .}$

Lead Diversification: An example of how biotransformation studies proved critical in the expansion of the primary pharmacology SAR is evident from the work of Lall et al. (2020), where the authors exploited the complex biochemical reactions that can be performed by oxidizing enzymes for diversification of hit/lead chemical series. Original small molecule hits/leads were incubated with a battery of recombinant mammalian and bacterial CYP enzymes, liver microsomes, or microbes to biochemically generate mixtures of products in a single step that would otherwise be very labor-intensive to prepare by traditional synthetic chemistry approaches. Electrochemical methods and biomimetic metalloporphyrin oxidation catalysts were also employed to chemically synthesize mixtures of structurally diverse products. Using the antihistamine loratadine as an example, over 10 unique products were generated from a combination of biochemical and chemical methods. These products were isolated in pure form and initially characterized via ultrahigh performance liquid chromatography-MS, followed by an unambiguous determination of structures and concentrations (in solution) by microcryoprobe 
quantitative NMR (Walker et al., 2014). The isolated compounds were subjected to in vitro pharmacological testing and also yielded sufficient product for in vitro assessment of relevant ADME parameters such as passive permeability and hepatic $\mathrm{CL}_{\text {int }}$. In vitro pharmacological (histamine $\mathrm{H} 1$ receptor agonist activity) testing of the biochemically/chemically generated loratadine analogues revealed that four possessed greater affinity for the human histamine $\mathrm{H}_{1}$ receptor, while five analogues showed a less than two-fold decrease in affinity, relative to loratadine. The availability of in vitro pharmacology and ADME data can allow a drug discovery team to weigh the pros and cons for a given set of lead compounds generated by these methods, and subsequently decide whether to invest in the scaling-up of compound synthesis for further testing. This work represents a novel role for traditional biotransformation scientists, whose function historically has been to characterize the metabolism of lead compounds, not to generate new ones. It also exemplifies how the synergy of organic chemistry, analytical chemistry, and biochemistry (all highly-valued skill sets for a biotransformation scientist) in small molecule lead diversification benefits teams by allowing them to rapidly probe previously unexplored chemical space.

\section{Drug development}

As investigational drug candidates progress through the various phases of clinical development, a thorough characterization of their metabolic pathways in animals and humans, with detailed knowledge of the drug metabolizing enzymes responsible for the metabolic elimination in humans, is required to address the potential for pharmacokinetic drug interactions and toxicological safety concerns. Given the regulatory importance of these studies, human ADME/mass balance activities are generally considered to be an important responsibility of a biotransformation scientist in medium-to-large pharmaceutical companies. 
Characterization of metabolites and metabolic pathways in the development phase is an extension of the fit-for-purpose metabolism work conducted with unlabeled compounds in the discovery phase to support candidate nomination. Studies in the development phase are usually conducted with radiolabeled $\left({ }^{3} \mathrm{H}\right.$ or $\left.{ }^{14} \mathrm{C}\right)$ compounds in in vitro systems (e.g., liver microsomes and hepatocytes from human and animals used for safety studies), and in vivo matrices (plasma, urine, and feces) following administration of the radiolabeled compound to animals and humans as part of mass balance/metabolite identification studies needed for regulatory filing for a new therapeutic agent (Penner et al., 2009; White et al., 2013). Metabolism studies with radiolabeled material enable the quantitative assessment of metabolites that are formed under in vitro conditions, as well as in vivo (e.g., metabolites in circulation in plasma or present in excreta from animals and humans). Biotransformation scientists also assist radiochemists in identifying metabolically stable regions for incorporation of the radiolabel, since they possess a working knowledge on the metabolically labile sites of an investigational drug, which is acquired during the discovery phase.

Characterization of metabolites in biological fluids, particularly in circulation, require that a biotransformation/metabolism scientist possess appropriate analytical skills (operation of LCMS/MS instrumentation), and is well-versed in the interpretation of mass spectral fragmentation patterns and potentially one- and two-dimensional NMR $\left({ }^{1} \mathrm{H}\right.$ or $\left.{ }^{14} \mathrm{C}\right)$ data to elucidate metabolite structures. While most metabolites can be (or are) identified in a discovery setting, there will be instances where disproportionate (metabolite area-under-the-plasma concentration vs. time curve exceeding $10 \%$ of the total drug-related material) or potentially human-unique metabolites are detected in human plasma, and will require subsequent qualification in plasma samples from animal toxicology studies as per the metabolites in safety testing (MIST) guidance issued by the 
regulatory agencies (Schadt et al., 2018). In the case of major metabolites (observed in vitro incubations and/or in circulation in animals and human), particularly ones observed from oxidative metabolism, accessing their corresponding authentic synthetic standards is important, since it allows additional insights into their contribution to the primary pharmacology (biologically active metabolites) as well as off-target (change in selectivity against receptor or enzyme sub-types, undesired activity for human ether-a-go-go gene product and other ion channels, etc.) and drug-drug interaction (potential to inhibit or induce human drug metabolizing enzymes such as CYPs) profiles (Yeung et al., 2011; Yu and Tweedie 2013; Yu et al., 2015). While most oxidative metabolite standards can be synthesized by medicinal chemists once the structure has been established, there will be instances where synthesis may prove difficult due to regiochemical or steric considerations in the vicinity of the site prone to oxidation. In such cases, authentic metabolite standards can also be generated by biotransformation scientists through scaled-up incubations of the parent compounds in matrices such as microsomes or hepatocytes. The enzymatically generated metabolites are isolated and purified by preparative HPLC, and are unambiguously characterized by LC-MS/MS and often times in a quantitative fashion utilizing NMR spectroscopy (Walker et al., 2014).

Human Mass Balance/ADME Studies: Metabolism studies on ozanimod, a potent sphingosine 1phosphate antagonist recently approved to treat relapsing multiple sclerosis and ulcerative colitis, illustrate the importance of biotransformation science in the development space. Preliminary metabolite identification studies on unlabeled ozanimod in a discovery setting led to the identification of three metabolites (RP101988, RP101075 and RP101442, Figure 3), all of which retained primary pharmacology. Rate-limiting steps in the formation of the three metabolites were mediated via CYP and a combination of alcohol dehydrogenase/aldehyde dehydrogenase 
$(\mathrm{ADH} / \mathrm{ALDH})$ enzymes. However, a subsequent human mass balance/ADME study with ${ }^{14} \mathrm{C}-$ ozanimod (1 mg) revealed a more complex picture (Surapaneni et al., 2021). Ozanimod was extensively metabolized via de novo metabolic pathways catalyzed by multiple non-CYP enzymes, including MAO B, carbonyl reductases (CBR), aldo-keto reductase (AKR), $\beta$ hydroxysteroid dehydrogenases ( $\beta$-HSD) (Figure 3) (Bai et al., 2021; Surapaneni et al., 2021). Moreover, ozanimod also underwent reductive metabolism in the gut microflora, which led to cleavage of its oxadiazole ring and the eventual formation of the corresponding benzoic acid metabolite (RP101124) (Surapaneni et al., 2021).

Of particular interest were the circulating metabolites of ozanimod. The plasma profile revealed the presence of two major circulating metabolites, an active indanone derivative (CC112273) and the inactive benzoic acid metabolite RP101124, which collectively constituted $\sim 50 \%$ of the total circulating radioactivity. Metabolite CC112273 accounted for $33 \%$ of radioactivity after a single oral dose of ${ }^{14} \mathrm{C}$-ozanimod and more importantly, had a long circulating $\mathrm{t}_{1 / 2}$ of $\sim 10$ days. Thus, following multiple oral doses, CC112273 accumulated leading to $\sim 73 \%$ of the total active drug exposure on the final day of dosing. Furthermore, comparison of CC112273 exposures in human and preclinical species used for toxicological assessments suggested significant interspecies differences in its formation/elimination and led to MIST issues that had to be resolved. Given the disproportionate nature of its formation in humans, CC112273 warranted further characterization with regards to its origins. Systematic enzyme reaction phenotyping studies revealed that $\mathrm{CC} 112273$ was formed sequentially via conversion of ozanimod to the indaneamine metabolite, RP101075 via CYP3A4-mediated $N$-dealkylation of the hydroxyethyl side chain followed by subsequent MAO B catalyzed oxidative deamination leading to the corresponding indanone. To characterize the involvement of gut microflora in the 
formation of RP101124, ozanimod was incubated with fresh rat fecal material collected from vehicle dosed rats or those that were treated with antibiotics under anerobic conditions. The lack of RP101124 in feces from antibiotic treated rats confirmed the role of gut bacteria in its formation. Another circulating metabolite worth mentioning was the indanol derivative, CC1084037. Although this metabolite accounted for $\sim 6 \%$ of the circulating radioactivity following a single oral dose of ozanimod to humans, the metabolite was involved in the redox cycling with equilibrium in favor of CC112273. Systematic studies using inhibitors and recombinant enzymes indicated that $\mathrm{CBR}$ was the primary enzyme, which catalyzed the reduction of CC112273 to CC1084037. Further assessment of disposition of CC1084037 suggested that this metabolite was rapidly oxidized in human hepatocytes and human liver subcellular fractions (cytosol and microsomes) and this oxidation was catalyzed by non-CYPs,

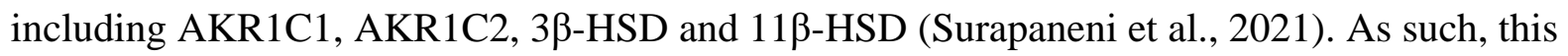
case study exemplifies the need to conduct human ADME studies in a timely fashion to assess mass balance and quantitatively identify metabolites to assess compliance with MIST. In addition, the complex metabolism observed with ozanimod emphasizes the importance of indepth knowledge in the areas of organic chemistry and enzymology that are required for biotransformation studies in the development space.

Preclinical Safety Studies: The importance of a biotransformation scientist's skills within drug development can additionally be illustrated by a case study with Empagliflozin (Jardiance®). Empagliflozin (Figure 4) is a selective inhibitor of the sodium-dependent glucose transporter 2 currently approved for the treatment of patients with Type 2 diabetes mellitus and cardiovascular disease. During late-stage development of empagliflozin, results from a 2-year carcinogenicity study in CD-1 mice indicated a statistically significant increase in the incidence of renal tubular 
adenomas and carcinomas in only the male mice at the highest dose level (1,000 mg/kg/day).

Several company-wide strategies were employed to investigate the mechanism and causality of carcinogenesis, including a DMPK-based ADME and biotransformation approach to interrogate the localized carcinogenic finding within the kidneys of male mice. (Taub et al., 2015)

Following administration of $1,000 \mathrm{mg} / \mathrm{kg}\left[{ }^{14} \mathrm{C}\right]$ empagliflozin to male and female $\mathrm{CD}-1 \mathrm{mice}$, plasma exposure and fecal excretion of empagliflozin did not display sex-based differences; however, empagliflozin urinary excretion was $\sim 12$-fold higher in female mice compared to male mice. This phenomenon provided the first clue leading to the hypothesis that sex-based differences in empagliflozin clearance could be a determining factor in formation of renal tumors in male mice. Subsequently, several in vitro studies were conducted to investigate the potential for sex-specific differences in empagliflozin metabolism utilizing male and female CD-1 mouse kidney microsomes. A cyclic hemiacetal metabolite (referred to as M466/2, Figure 4) was predominantly formed in males by a factor of 22-fold (Figure 5) compared to females. Cyclic hemiacetal structures are inherently unstable and are in equilibrium with their corresponding aldehyde forms. Although not the predominant isomer in equilibrium, the M466/2 aldehyde was hypothesized to undergo a base or acid catalyzed retro-Michael addition to produce the corresponding phenol metabolite (M380) and 4-hydroxycrotonaldehyde (4-OH-CTA) (Figure 4). 4-OH-CTA is a Michael acceptor which reacts with nucleophilic sites on DNA and proteins, leading to covalent adduct formation and toxicity. Key metabolite trapping studies with $\left[{ }^{3} \mathrm{H}\right] \mathrm{GSH}$, demonstrated the formation of the 4-OH-CTA- $\left[{ }^{3} \mathrm{H}\right] \mathrm{GSH}$ adduct, thus providing credence to the reactive metabolite hypothesis and rationale for the carcinogenicity observed only in male mouse kidneys. Moreover, 4-OH-CTA was shown to be cytotoxic to mouse renal tubule cells in vitro and is associated with renal injury, notably karyomegaly and single cell 
necrosis (Smith et al., 2017). From this case example, a foundational knowledge of drug metabolism, biotransformation, and organic chemistry led to the discovery and root cause of carcinogenicity that was specifically identified in male mice kidneys.

\section{Biotransformation Studies to Support Development of New Modalities.}

While embracing the evolving therapeutic landscape, biotransformation scientists have expanded their roles and responsibilities beyond conventional small molecules. These extended roles are the result of the increasing interest in understanding biotransformation of new modalities such as PROTACs, peptides, oligonucleotides, antibodies, proteins, and antibody-drug conjugates (ADCs). Similar to conventional small molecule drugs, medium to large molecules are also subject to biotransformation, resulting in catabolites/metabolites that affect clearance, pharmacology, toxicology, and increase the immunogenic potential. Common biotransformation pathways of medium/large molecules include isomerization and glycation of proteins and antibodies, oxidation of peptides, proteins, and antibodies, deamidation and truncation/clipping of peptides, proteins, and oligonucleotides, payload metabolism, and deconjugation of ADCs (He et al., 2017; Wei et al., 2018; Kaur et al., 2020; Weidolf et al., 2021). For example, givosiran, a small interfering RNA (siRNA) is susceptible to nucleases, glycosidases, and amidases which results in truncation and removal of GalNAc and loss of linkers, respectively (Li et al., 2021). Peptides can be susceptible to CYP oxidation (e.g., cyclosporin) and/or undergo hydrolysis (e.g., leuprorelin and cetrorelix) via peptidases (Jyrkas and Tolonen 2021). Proteins and antibodies can undergo isomerization (e.g., aspartic acid to isoaspartic acid) and deamidation (e.g., conversion of asparagine to a mixture of aspartic acid and isoaspartic acid), in addition to the typical hydrolytic process. These degradation reactions can raise concerns if the liability is in the complementary determining regions and impacts the binding affinity and biological activity 
against the target (Vlasak et al., 2009; Bults et al., 2016). ADC biotransformation including payload metabolism, linker deconjugation, and sequential payload release has played a key role in guiding ADC design and optimization (Ma et al., 2016; Su et al., 2016; Su et al., 2018; Zhang et al., 2018). Optimization of ADCs aims to reduce undesired payload metabolism and linker deconjugation in circulation and improve effective payload release following linker deconjugation/immolation in tumors (Su et al., 2021).

Given the distinct features of larger molecules compared to small molecules, different expertise and techniques are required for biotransformation scientists to perform large molecule biotransformation studies. Knowledge of nucleases and peptidases is the foundation for understanding biotransformation of large molecules. In order to eliminate signal interference from biological matrices (e.g., proteins in plasma and tissue), biotransformation specialists spend considerable effort optimizing sample extraction conditions. Protein precipitation and solid phase extraction are often used for peptides and oligonucleotides while immunoaffinity purification is favored for proteins, antibodies, and ADCs (Fung et al., 2016; Donnelly et al., 2019). Having an expertise in separation science using HPLC or capillary electrophoresis, which can be coupled with mass spectrometry for biotransformation work, is also beneficial in this field (Han et al., 2021). High resolution MS is highly recommended for elucidating complex biotransformation and sites of metabolism of large molecules (Wagner-Rousset et al., 2014; Su et al., 2016; Su et al., 2018). LC-MS/MS methods are typically used for accurate quantitation of catabolites/metabolites (Bults et al., 2016; Lui et al., 2018; Wei et al., 2018)

A noteworthy example of leveraging the utility of biotransformation insights to guide science in the disposition optimization of large molecule modalities is evident in the ADC field. Biotransformation studies play a critical role in understanding the mechanism of action and 
$\mathrm{PK} / \mathrm{PD}$ relationships of ADCs and the challenge of asymmetric disposition in plasma vs. tumor tissue given the target-mediated uptake nature and different metabolizing enzymes in plasma and tumor cells. Therefore, it is important to identify the relevant drug-related analyte to rationalize efficacy profiles. For example, two close analogs of pyrrolo[2,1-c][1,4]benzodiazepine-dimer (PBD) site-specific THIOMAB ${ }^{\mathrm{TM}}$ ADCs with cyclobutyl (ADC 1) versus cyclopropyl (ADC 2) hindered disulfide linkers (Figure 6) showed dramatic difference in efficacy in mice (Ma et al., 2016). ADC 1 was efficacious in mice while ADC 2 was not despite similar stability of the two ADCs in circulation. Detailed examination of the tumor tissue samples revealed that that ADC 1 resulted in effective intratumoral payload (free PBD dimer) concentration while ADC 2 produced only the inactive linker-payload that did not bind to DNA. In vitro mechanistic studies revealed that effective payload release from ADC 1 occurred through self-immolation following linker cleavage, which was in contrast to the lack of self-immolation or payload release from ADC 2. In this case study, biotransformation studies were critical in identifying the relevant analyte intratumoral payload and quantitative measure of the concentrations provided insights into the pharmacokinetics/pharmacodynamics, which rationalized the observed efficacy profiles (Zhang et al., 2018). Consequently, a comprehensive strategy of balancing ADC stability in circulation and payload release in tumor was established for optimal ADC design and efficacy on the basis of linker-payload driven metabolism insights.

\section{Career Options for Biotransformation Scientists in the Pharmaceutical}

\section{Industry}

Medium-to-large pharmaceutical organizations. In the early stages of their industrial careers, most biotransformation scientists are involved in the design, execution, interpretation, and communication of metabolite identification data from in vitro (or in vivo) matrices in support of 
both conventional small molecule therapeutics and large molecule modalities. Since information pertaining to the metabolism of chemical hits/leads is incorporated in the medicinal chemistry design process, specialized biotransformation scientists closely align with DMPK counterparts and medicinal chemists to support drug discovery projects. As new hires, scientists also acquire new skills (e.g., operation of state-of-the-art bioanalytical instrumentation, biosynthesis of metabolites, isotope labeling experiments, etc.) to support their routine activities, and gain familiarity with the organizational setup of the DMPK department and the discovery/development organization at large. With many companies showing an increased trend in the outsourcing of research and development activities, an array of new responsibilities for the biotransformation scientist has emerged that include interfacing with $\mathrm{CRO}$ partners to design outsourced metabolite identification studies and interpret and review resulting data.

Career progression within medium-large companies for scientists with biotransformation expertise can follow multiple paths. Some choose to remain in their original trajectory, which specifically focuses on their biotransformation expertise. This path can lead to a role focused on providing biotransformation expertise within the organization, through an increased amount of time spent consulting with project teams on issues pertaining to drug metabolism, albeit likely with some reduction in time spent in the laboratory. Others may choose to pursue management responsibilities where structuring operations within the group and training new hires become a priority. A third path is the transition from a specialized biotransformation scientist role to a generalist role of a "drug hunter" by serving as the DMPK representative on drug discovery and development projects. The role and responsibilities of a DMPK “drug hunter” are elaborated on below. 
Small pharmaceutical or biotechnology companies. Small biotechnology companies (including academic drug discovery centers) with limited resources (financial and personnel) typically have minimal on-site laboratory capabilities to support research and development efforts. Thus, many lab-based activities, including DMPK efforts, are outsourced to CROs. To support this model, small companies tend to hire DMPK scientists with considerable amounts of relevant industry experience and a more generalized background in drug discovery to oversee all DMPK activities and carry out the roles and responsibilities of a DMPK "drug hunter".

Biotransformation Opportunities at CROs. Owing to the rapid expansion of CROs, employment opportunities which are "science facing" are widespread and vary in the pre-requisite experience required. These may be based in scientific operations, quality assurance or client services.

Furthermore, CROs have earned a reputation as employers of new graduates, and often have structured scientific graduate progression schemes to attract and nurture talent. The historical stance that a position within a CRO was often regarded as a steppingstone to another job is now obsolete; indeed many individuals have switched from careers in "large Pharma" to CROs where they also enjoy long and fulfilling careers within the industry.

While the general role of biotransformation scientists in pharmaceutical companies and CROs is largely similar, one key difference is that the CRO may not have access to complete datasets (e.g., supporting information if additional work has been done elsewhere). Thus, the ability to problem solve and innovate with restricted information and present elegant solutions provides opportunity for ingenuity. The largely client agnostic nature of contract research raises a unique point, particularly in the field of biotransformation: CRO scientists are exposed to a large number and wide breadth of chemistries from a diversity of clients and industries. Setting aside contractual limitations related to intellectual property and competitor non-disclosure, CRO 
scientists can leverage learnings from their exposure to these diverse chemical entities to accelerate project advancement. As such, CRO-based biotransformation scientists will also be exposed to the exact same repertoire of tools (e.g., biochemical matrices), tactics (e.g., chemical derivatization and isotope labeling to elucidate metabolite structures) and instrumentation (e.g. LC-MS/MS, NMR, etc.) as their large pharma counterparts towards the pursuit of drug metabolism studies. By combining an understanding of reaction mechanisms with the ability to interpret complex analytical datasets, the biotransformation chemist in a CRO can also be expected to present these data to a variety of audiences including colleagues, clients, internal partners, and regulatory authorities.

Venturing into a "Drug Hunter" Role with a Biotransformation Background. The dramatic reduction in clinical candidate attrition rates due to inadequate pharmacokinetics (Kola and Landis, 2004), which was largely achieved via the embedment of DMPK science/scientists in the early discovery process, is now a permanent fixture in virtually all pharmaceutical companies including start-up ventures. Consequently, an alternative career path evolved for scientists with medicinal chemistry/chemical toxicology background in the discovery DMPK realm as a "drug hunter" which exists in pharmaceutical companies and CROs of any size. In this role, DMPK scientists work in close collaboration with their counterparts from medicinal chemistry, pharmacology, toxicology, formulation sciences, etc. to support the progression of discovery projects from exploratory $\rightarrow$ lead development $\rightarrow$ clinical candidate identification/nomination, and hence the endowment of the title of "drug hunter". Post-candidate nomination, several organizations have installed an operational model where the DMPK project representative continues to serve on the clinical development team. Thus, in addition to their drug discovery skills, discovery DMPK scientists also gain considerable exposure and hands-on experience 
(e.g., the preparation of regulatory ADME documents in support of first-in-human clinical studies, etc.) in the development sphere, where they work hand-in-hand with representatives from clinical pharmacokinetics and clinical sciences groups, regulatory personnel, etc.

Ultimately, what this translates to, is a well-rounded DMPK scientist with experience in both the discovery and development space.

Unlike the role of a biotransformation scientist in discovery described above, the role of the “drug hunter” DMPK scientist is not laboratory based. Rather, they are scientific thinkers with the ability to integrate ADME data for new chemical entities from multiple sources, which is then used to scientifically justify (or deny) the progression of chemical hits/leads into viable clinical candidates. Because this activity requires a fair degree of complex data interpretation, presentation of ADME-related challenges, and appropriate risk mitigation strategies to project team members with diverse academic backgrounds (some with little to no understanding of ADME sciences), it is important that discovery DMPK scientists are efficient communicators of their science to a broad audience. Much of these "soft skills" are not directly taught in graduate schools; some individuals naturally possess these skills, while others master them over the years on the job.

Having sound organic chemistry concepts instilled during graduate school training, with hands-on training in medicinal chemistry and/or chemical toxicology is an important weapon in the arsenal of a "drug hunter" for several reasons. Like their counterparts in biotransformation roles, DMPK scientists in the "drug hunter" roles can use their knowledge to predict metabolic soft spots, likely enzymes responsible for metabolism, and potentially active metabolites. However, to ensure a successful career in the drug discovery paradigm, chemistry-trained DMPK scientists will require adequate amount of training across multiple disciplines (e.g., use of 
physicochemical parameters in medicinal chemistry design, high-throughput primary and secondary pharmacology screening, in vivo animal models of pharmacology, drug safety concepts, projection of human pharmacokinetics, etc.), and most importantly, in DMPK areas other than drug metabolism and biotransformation science. Amongst these, a fundamental understanding of pharmacokinetics concepts and related parameters is most essential since a considerable effort in preclinical drug discovery involves animal pharmacokinetics studies to support in vivo pharmacology and drug safety testing. Establishing a firm knowledge base on concepts pertaining to oral absorption (e.g., solubility, passive permeability, efflux, and first pass metabolism across the intestinal tract and liver) and distribution (e.g., plasma protein binding) is an equally important activity, which is needed to rationalize the output from preclinical pharmacokinetics studies.

Consequently, scientists with biotransformation training hired directly from graduate education into these roles or those who work in a lab-based position before acquiring these roles will be required to undergo extensive training to master these skills "on the job", usually via tutelage from senior colleagues and/or courses offered within companies or universities, specializing in pharmacokinetics. Historically, scientists with a strong academic background in pharmacokinetics and/or quantitative pharmacology disciplines have been generally preferred for the role of a discovery DMPK scientist, given the predictive nature of the science in this domain. Suffice it to say, individuals with a pharmacokinetics background have an equally steep learning curve mastering the concepts of organic chemistry and biotransformation/drug metabolism science as applicable to the drug discovery process.

External disclosure of research work at all stages of the drug discovery/development process is generally encouraged in all pharmaceutical companies since such activities provides external 
visibility for the scientific staff as well as the department and the company. Towards this end, biotransformation scientists in the development arena can have an edge over their discovery counterparts with regards to an opportunity to present and/or publish their research (e.g., disclosure of human and animal mass balance/metabolism studies) on investigational drug candidates, particularly those in advanced clinical development. Timely disclosure of preclinical discovery work (e.g., de novo medicinal chemistry tactics to resolve ADME issues) by discovery biotransformation scientists is contingent upon the initial disclosure of relevant intellectual property (e.g., filing of patents) surrounding the chemical matter and/or initial medicinal chemistry publications detailing pharmacology structure-activity relationships.

\section{Challenges, Opportunities, and Outlook for Future Training of}

\section{Biotransformation Scientists}

Over the last few decades, a steady decline in the number of chemistry (or medicinal chemistry) departments in the United States (and globally) who trained those within the existing biotransformation community presents a challenge that will hinder recruitment of the future cohort of biotransformation scientists. The decline is partially due to shifts in research direction, lack of government grants/funding, and the retirement of several prominent faculty in the field who are considered pioneers of biotransformation science (MacCoss and Baillie, 2004). To add to this dilemma, biotransformation science has been inaccurately classified as a "mature" field with limited growth potential, and consequently, the projected need for training in this area diminished. The corresponding thought process that evolved is that academic departments would be best served by diversifying research training and reducing investment in biotransformation science. 
Currently, the need for biotransformation scientists at all levels within the pharmaceutical industry is strong and a limited candidate pool results in competition to hire trained candidates. This need is further evident from an examination of recent drug approval trends in the US. For example, between 2016-2020, 228 novel therapeutics were approved by the US Food and Drug Administration, with conventional small molecule therapeutics constituting the majority (146, $64 \%$ ) of the new approvals. The remainder of new approvals comprised of monoclonal antibodies $(42,18 \%)$, peptides $(14,6 \%)$, oligonucleotides $(10,4 \%)$ ADCs, $(6,3 \%)$, and other types of protein drugs (enzymes, fusion proteins, etc.) (10, 4\%) (De La Torre and Albericio 2017; 2018 De La Torre and Albericio 2020;2021). Moreover, examination of recent literature and press releases reveal clinical trials with several newer modalities such as protein degraders (Qi et al. 2021). Such new paradigm shifts from conventional small molecule therapeutics to the increasing use of peptides, ADCs, protein degraders, etc. has also opened new avenues for the next generation of biotransformation scientists who specialize in the biochemistry/enzymology, metabolism, and bioanalysis of complex modalities (e.g., peptides, oligonucleotides, etc.) during their graduate training. As an industry, we must commit to investigating the metabolism of these newer modalities with the high level of rigor that we apply to small molecules to ensure the continued development of safe and effective therapeutics.

It is still, and will always be, possible to train biotransformation scientists in academia but it is increasingly challenging. Three systemic challenges contribute to this difficulty; the lack of support for funding purely drug metabolism or biotransformation research, the dearth of suitable NIH study sections with the expertise to review biotransformation-related grants, and the lack of commitment from federal institutes who consider biotransformation research more fit for industrial purposes than academia. To overcome some of these challenges with funding agencies, 
biotransformation aims can be embedded as part of a larger project to investigate toxicity or health related drug concepts without being the main focus. For example, if the main focus of the study is to investigate the cardiotoxicity of tyrosine kinase inhibitors, then metabolism/bioactivation of tyrosine kinase inhibitors can be a sub-aim that will provide opportunities for trainees to study drug biotransformation. Another alternative could be a collaboration between industry DMPK groups and academic laboratories, wherein industry funds graduate students or post-doctoral fellows to work on topics of interest to the drug metabolism community. Such an activity could serve as fertile ground for training pre- and post-doctoral students in biotransformation science that benefits both parties. These opportunities are limited and are more common in departments and schools with close personal ties to industry. There is potential, however, to foster more of these collaborations through scientific societies such as ISSX.

Another strategy for training is through engaging advanced undergraduate students in biotransformation research projects to motivate future interest in the field. Including drug metabolism in undergraduate chemistry or biochemistry curricula, providing guidance toward doctoral level training in the field, and encouraging exposure to industry DMPK scientists can all help garner future interest in biotransformation. While we do not anticipate the creation or conversion of new academic departments that are dedicated to biotransformation research, there are ample opportunities for interested students to carry undergraduate thesis research at various universities in the US and around the world. As we consider the heightened need for scientists with advanced training in biotransformation, especially with the emergence and prominence of new therapeutic modalities, future opportunities and employment outlook indicate the vital need to promote training and development within the biotransformation field. 
DMD-MR-2021-000658

\section{Author Contributions}

Participated in research design: Kramlinger, Dalvie, Heck, Kalgutkar, O’Neill, Su, Teitelbaum,

Totah

Conducted experiments: N/A

Contributed new reagents or analytic tools: N/A

Performed data interpretation: N/A

Contributed to the writing of the manuscript: Kramlinger, Dalvie, Heck, Kalgutkar, O'Neill, Su, Teitelbaum, Totah

\section{Footnotes}

The views expressed in the manuscript are solely those of the authors and do not represent the views of the companies and institutions they are affiliated with.

The authors declare that they have no conflicts of interest with the contents of this article.

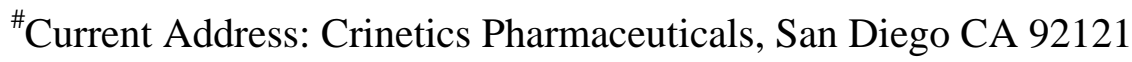

${ }^{\%}$ Current address: Drug Metabolism and Pharmacokinetics, Mersana Therapeutics Inc., 840

Memorial Dr, Cambridge, MA 02139 
DMD-MR-2021-000658

\section{References}

Bai A, Shanmugasundaram V, Selkirk JV, Surapaneni S, and Dalvie D (2021) Investigation into MAO B-mediated formation of CC112273, a major circulating metabolite of ozanimod in humans and preclinical species: stereospecific oxidative deamination of (S)enantiomer of indaneamine (RP101075) by MAO B. Drug Metab Dispos 49: 601-609.

Bults PR, Bischoff R, Bakker H, Gietema JA, and van de Merbel NC (2016) LC-MS/MS-Based Monitoring of In Vivo Protein Biotransformation: Quantitative Determination of Trastuzumab and Its Deamidation Products in Human Plasma. Anal Chem 88:18711877.

Cerny MA, Kalgutkar AS, Obach RS, Sharma R, Spracklin DK, and Walker GS (2020) Effective application of metabolite profiling in drug design and discovery. J Med Chem 63:63876406.

De La Torre BG and Albericio F (2017) The pharmaceutical industry in 2016. An analysis of FDA drug approval from a perspective of the molecule type. Molecules 22:368.

De La Torre BG and Albericio F (2018) The pharmaceutical industry in 2017. An analysis of FDA drug approvals from the perspective of molecules. Molecules 23:533.

De La Torre BG and Albericio F(2020) The pharmaceutical industry in 2019. An analysis of FDA drug approvals from the perspective of molecules. Molecules 25:745.

De LaTorre BG and Albericio F (2021) The pharmaceutical industry in 2020. An analysis of FDA drug approval from a perspective of the molecules. Molecules 26:627.

Di L, Keefer C, Scott DO, Strelevitz TJ, Chang G, Bi YA, Lai Y, Duckworth J, Fenner K, Troutman MD, and Obach RS (2012) Mechanistic insights from comparing intrinsic 
clearance values between human liver microsomes and hepatocytes to guide drug design. Eur J Med Chem 57:441-448.

Donnelly DP, Rawlins CM, DeHart CJ, Fornelli L, Schachner LF, Lin Z, Lippens JL, Aluri KC, Sarin R, Chen B, Lantz C, Jung W, Johnson KR, Koller A, Wolff JJ, Campuzano IDG, Auclair JR, Ivanov AR, Whitelegge JP, Paša-Tolić L, Chamot-Rooke J, Danis PO, Smith LM, Tsybin YO, Loo JA, Ge Y, Kelleher NL, and Agar JN (2019) Best practices and benchmarks for intact protein analysis for top-down mass spectrometry. Nat Methods 16:587-594.

Fung EN, Bryan P, and Kozhich A (2016) Techniques for quantitative LC-MS/MS analysis of protein therapeutics: advances in enzyme digestion and immunocapture. Bioanalysis 8:847-856.

Han M, Wang Y, Cook K, Bala N, Soto M, Rock DA, Pearson JT, and Rock BM (2021) Universal Automated Immunoaffinity Purification-CE-MS Platform for Accelerating Next Generation Biologic Design. Anal Chem 93:5562-5569.

He J, Su D, Ng C, Liu L, Yu SF, Pillow TH, Del Rosario G, Darwish M, Lee BC, Ohri R, Zhou H, Wang X, Lu J, Kaur S, and Xu K (2017) High-Resolution Accurate-Mass Mass Spectrometry Enabling In-Depth Characterization of in Vivo Biotransformations for Intact Antibody-Drug Conjugates. Anal Chem 89:5476-5483.

Hosea NA, Collard WT, Cole S, Maurer TS, Fang RX, Jones H, Kakar SM, Nakai Y, Smith BJ, Webster R, and Beaumont K (2009) Prediction of human pharmacokinetics from preclinical information: comparative accuracy of quantitative prediction approaches. $J$ Clin Pharmacol 49:513-533. 


\section{DMD-MR-2021-000658}

Jyrkas J and Tolonen A (2021) Hepatic in vitro metabolism of peptides; Comparison of human liver S9, hepatocytes and Upcyte hepatocytes with cyclosporine A, leuprorelin, desmopressin and cetrorelix as model compounds. J Pharm Biomed Anal 196:113921.

Kalgutkar AS (2020) Designing around structural alerts in drug discovery. J Med Chem 63:62766302.

Kaur S, Bateman KP, Glick J, Jairaj M, Kellie F, Sydor J, and Zeng J (2020) IQ consortium perspective: complementary LBA and LC-MS in protein therapeutics bioanalysis and biotransformation assessment. Bioanalysis 12:257-270.

Kola I and Landis J (2004) Can the pharmaceutical industry reduce attrition rates? Nat Rev Drug Discov 3:711-715.

Lall MS, Bassyouni A, Bradow J, Brown M, Bundesmann M, Chen J, Ciszewski G, Hagen AE, Hyek D, Jenkinson S, Liu B, Obach RS, Pan S, Reilly U, Sach N, Smaltz DJ, Spracklin DK, Starr J, Wagenaar M, and Walker GS (2020) Late-stage lead diversification coupled with quantitative nuclear magnetic resonance spectroscopy to identify new structureactivity relationship vectors at nanomole-scale synthesis: application to loratadine, a human histamine H1 receptor inverse agonist. J Med Chem 63:7268-7292.

Li J, Liu J, Zhang X, Clausen V, Tran C, Arciprete M, Wang Q, Rocca C, Guan LH, Zhang G, Najarian D, Xu Y, Smith P, Wu JT, and Chong S (2021) Nonclinical Pharmacokinetics and Absorption, Distribution, Metabolism, and Excretion of Givosiran, the First Approved N-Acetylgalactosamine-Conjugated RNA Interference Therapeutic. Drug Metab Dispos 49:572-580. 
Lin JH and Lu AY (1997) Role of pharmacokinetics and metabolism in drug discovery and development. Pharmacol Rev 49:403-449.

Liu L, Xu K, Li J, Maia M, Mathieu M, Elliot R, Yang J, Nijem I, Kaur S (2018) Optimizing hybrid LC-MS/MS binding conditions is critical: impact of biotransformation on quantification of trastuzumab. Bioanalysis 10: 1819-1831.

Ma Y, Khojasteh SC, Hop CE, Erickson HK, Polson A, Pillow TH, Yu SF, Wang H, Dragovich PS, and Zhang D (2016) Antibody Drug Conjugates Differentiate Uptake and DNA Alkylation of Pyrrolobenzodiazepines in Tumors from Organs of Xenograft Mice. Drug Metab Dispos 44:1958-1962.

MacCoss M and Baillie TA (2004) Organic Chemistry in Drug Discovery. Science 303:1810-1813.

Park BK, Boobis A, Clarke S, Goldring CE, Jones D, Kenna JG, Lambert C, Laverty HG, Naisbitt DJ, Nelson S, Nicoll-Griffith DA, Obach RS, Routledge P, Smith DA, Tweedie DJ, Vermeulen N, Williams DP, Wilson ID, and Baillie TA (2011) Managing the challenge of chemically reactive metabolites in drug development. Nat Rev Drug Discov 10:292-306.

Penner N, Klunk LJ, and Prakash C (2009) Human radiolabeled mass balance studies: objectives, utilities and limitations. Biopharm Drug Dispos 30:185-203.

Qi SM, Dong J, Xu ZY, Cheng XD, Zhang WD, Qin JJ (2021) PROTAC: an effective targeted protein degradation strategy for cancer therapy. Front Pharmacol 12:692574.

Schadt S, Bister B, Chowdhury SK, Funk C, Hop CECA, Humphreys WG, Igarashi F, James AD, Kagan M, Khojasteh SC, Nedderman ANR, Prakash C, Runge F, Scheible H, Spracklin DK, Swart P, Tse S, Yuan J, and Obach RS (2018) A decade in the MIST: 


\section{DMD-MR-2021-000658}

Learnings from investigations of drug metabolites in drug development under the “Metabolites in Safety Testing” regulatory guidance. Drug Metab Dispos 46:865-878.

Shanu-Wilson J, Evans L, Wrigley S, Steele L, Atherton J, and Boer J (2020) Biotransformation: impact and application of metabolism in drug discovery. ACS Med Chem Lett 11:20872107.

Smith JD, Huang Z, Escobar PA, Foppiano P, Maw H, Loging W, Yu H, Phillips JA, Taub M, and Ku WW (2017) A Predominant Oxidative Renal Metabolite of Empagliflozin in Male Mice Is Cytotoxic in Mouse Renal Tubular Cells but not Genotoxic. Int J Toxicol 36:440-448.

Stepan AF, Karki K, McDonald WS, Dorff PH, Dutra JK, Dirico KJ, Won A, Subramanyam C, Efremov IV, O'Donnell CJ, Nolan CE, Becker SL, Pustilnik LR, Sneed B, Sun H, Lu Y, Robshaw AE, Riddell D, O'Sullivan TJ, Sibley E, Capetta S, Atchison K, Hallgren AJ, Miller E, Wood A, and Obach RS. (2011) Metabolism-directed design of oxetanecontaining arylsulfonamide derivaties as $\gamma$-secretase inhibitors. J Med Chem 54:77727783.

Su D, Ng C, Khosraviani M, Yu SF, Cosino E, Kaur S, and Xu K (2016) Custom-Designed Affinity Capture LC-MS F(ab')2 Assay for Biotransformation Assessment of SiteSpecific Antibody Drug Conjugates. Anal Chem 88:11340-11346.

Su D, Kozak KR, Sadowsky J, Yu SF, Fourie-O'Donohue A, Nelson C, Vandlen R, Ohri R, Liu L, Ng C, He J, Davis H, Lau J, Del Rosario G, Cosino E, Cruz-Chuh JD, Ma Y, Zhang D, Darwish M, Cai W, Chen C, Zhou H, Lu J, Liu Y, Kaur S, Xu K, and Pillow TH (2018) Modulating Antibody-Drug Conjugate Payload Metabolism by Conjugation Site and Linker Modification. Bioconjug Chem 29:1155-1167. 


\section{DMD-MR-2021-000658}

Su D and Zhang D (2021) Linker Design Impacts Antibody-Drug Conjugate Pharmacokinetics and Efficacy via Modulating the Stability and Payload Release Efficiency. Front Pharmacol 12:687926.

Surapaneni S, Yerramilli U, Bai A, Dalvie D, Brooks J, Wang X, Selkirk JV, Yan YG, Zhang P, Hargreaves R, Kumar G, Palmisano M, and Tran JQ (2021) Absorption, metabolism, and excretion, in vitro pharmacology, and clinical pharmacokinetics of ozanimod, a novel sphingosine 1-phosphate receptor modulator. Drug Metab Dispos 49:405-419.

Taub ME, Ludwig-Schwellinger E, Ishiguro N, Kishimoto W, Yu H, Wagner K, and Tweedie D (2015) Sex-, species-, and tissue-specific metabolism of empagliflozin in male mouse kidney forms an unstable hemiacetal metabolite (M466/2) that degrades to 4hydroxycrotonaldehyde, a reactive and cytotoxic species. Chem Res Toxicol 28:103-115.

Vlasak J, Bussat MC, Wang S, Wagner-Rousset E, Schaefer M, Klinguer-Hamour C, Kirchmeier M, Corvaia N, Ionescu R, and Beck A (2009) Identification and characterization of asparagine deamidation in the light chain CDR1 of a humanized IgG1 antibody. Anal Biochem 392:145-154.

Wagner-Rousset E, Janin-Bussat MC, Colas O, Excoffier M, Ayoub D, Haeuw JF, Rilatt I, Perez M, Corvaïa N, and Beck A (2014) Antibody-drug conjugate model fast characterization by LC-MS following IdeS proteolytic digestion. MAbs 6:273-285.

Walker DP, Bi FC, Kalgutkar AS, Bauman JN, Zhao SX, Soglia JR, Aspnes GE, Kung DW, Klug-McLeod J, Zawistoski MP, McGlynn MA, Oliver R, Dunn M, Li JC, Richter DT, Cooper BA, Kath JC, Hulford CA, Autry CL, Luzzio MJ, Ung EJ, Roberts WG, Bonnette PC, Buckbinder L, Mistry A, Griffor MC, Han S, and Guzman-Perez A (2008) 


\section{DMD-MR-2021-000658}

Trifluromethylpyrimidine-based inhibitors of proline-rich tyrosine kinase 2 (PYK2): structure-activity relationships and strategies for the elimination of reactive metabolite formation. Bioorg Med Chem Lett 18:6071-6077.

Walker GS, Bauman JN, Ryder TF, Smith EB, Spracklin DK, and Obach RS (2014)

Biosynthesis of drug metabolites and quantitation using NMR spectroscopy for use in pharmacologic and drug metabolism studies. Drug Metab Dispos 42:1627-1639.

Wei C, Su D, Wang J, Jian W, and Zhang D (2018) LC-MS Challenges in Characterizing and Quantifying Monoclonal Antibodies (mAb) and Antibody-Drug Conjugates (ADC) in Biological Samples. Current Pharmacology Reports 4:45-63.

Weidolf L, Björkbom A, Dahlén A, Elebring M, Gennemark P, Hölttä M, Janzén D, Li X, and Andersson S (2021). Distribution and biotransformation of therapeutic antisenseoligonucleotides and conjugates. Drug Discov Today 20:S1359-6446.

White RE, Evans DC, Hop CE, Moore DJ, Prakash C, Surapaneni S, and Tse FL (2013) Radiolabeled mass-balance excretion and metabolism studies in laboratory animals: a commentary on why they are still necessary. Xenobiotica 43:219-225.

Yeung CK, Fujioka Y, Hachad H, Levy RH, and Isoherranen N (2011) Are circulating metabolites important in drug-drug interactions? Quantitative analysis of risk prediction and inhibitory potency. Clin Pharmacol Ther 89:105-113.

Yu H and Tweedie D (2013) A perspective on the contribution of metabolites to drug-drug interaction potential: the need to consider both circulating levels and inhibition potency. Drug Metab Dispos 41:536-540. 
Yu H, Balani SK, Chen W, Cui D, He L, Humphreys WG, Mao J, Lai WG, Lee AJ, Lim HK, MacLauchlin C, Prakash C, Surapaneni S, Tse S, Upthagrove A, Walsky RL, Wen B, and Zeng Z (2015) Contribution of metabolites to P450 inhibition-based drug-drug interactions: scholarship from the drug metabolism leadership group of the innovation and quality consortium metabolite group. Drug Metab Dispos 43:620-630.

Zhang D, Yu SF, Khojasteh SC, Ma Y, Pillow TH, Sadowsky JD, Su D, Kozak KR, Xu K, Polson AG, Dragovich PS, and Hop CECA (2018) Intratumoral Payload Concentration Correlates with the Activity of Antibody-Drug Conjugates. Mol Cancer Ther 17:677685.

\section{Footnotes}

No external funding was used to support this manuscript. 


\section{DMD-MR-2021-000658}

\section{Figure Legends}

Figure 1. Optimization of metabolic $\mathrm{CL}_{\text {int }}$ for a series of $\mathrm{N}$-arylsulfonamide-based $\gamma$-secretase inhibitors. Adapted from Cerny et al. (2020).

Figure 2. Attenuating bioactivation liabilities with 5-aminooxindole-based PYK2 inhibitors. Adapted from Kalgutkar (2020).

Figure 3. Metabolism of ozanimod in humans. Adapted from Surapaneni et al. (2021).

Figure 4. Bioactivation pathway of Empagliflozin to 4-OH-CTA. Adapted from Taub et al. (2015).

Figure 5. Rate of M466/2 formation in kidney and liver microsomes from humans, CD-1 mice, and Wistar Han rats. Adapted from Taub et al. (2015).

Figure 6. Biotransformation of disulfide-linker containing ADCs. (A) Mechanisms of biotransformation of disulfide-linker containing ADCs including linker cleavage, selfimmolation and payload release in lysosome inside cell. (B) Differential biotransformation pathways resulting in free and active payload (PBD-dimer) released from cycobutyl thiol linkerpayload from ADC 1 that covalently binds to DNA but cyclopropyl thiol linker-payload from ADC 2 that does not bind to DNA. ADC 1: anti-CD22 cyclobutyl-containing disulfide PBD dimer ADCs; ADC 2: cyclopropyl-containing disulfide PBD dimer ADC. Adapted from Su and Zhang (2021). 


\section{Figure 1}

* marks the site of oxidative metabolism by CYP3A4

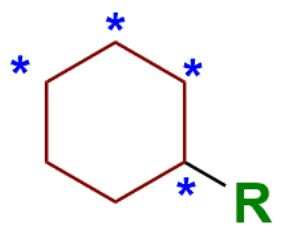

1

$\mathrm{HLM} \mathrm{CL}_{\text {int }}$ $(\mathrm{mL} / \mathrm{min} / \mathrm{kg})$

176

$>300$

2

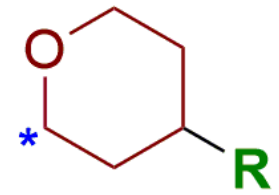

3

114 $\log D=5.6$

$I_{50}(A \beta 42, n M)=12$
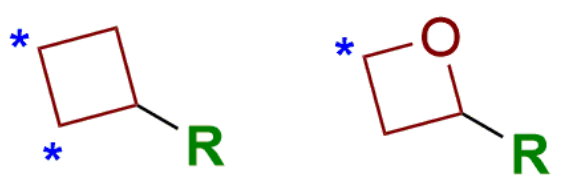

4

HLM CL int $_{\text {in }}$ $(\mathrm{mL} / \mathrm{min} / \mathrm{kg})$<smiles>[R]C1CCCCO1</smiles>

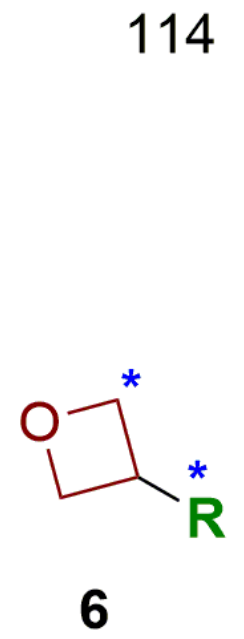

29

$I_{50}(A \beta 42, n M)=17$ 
Figure 3

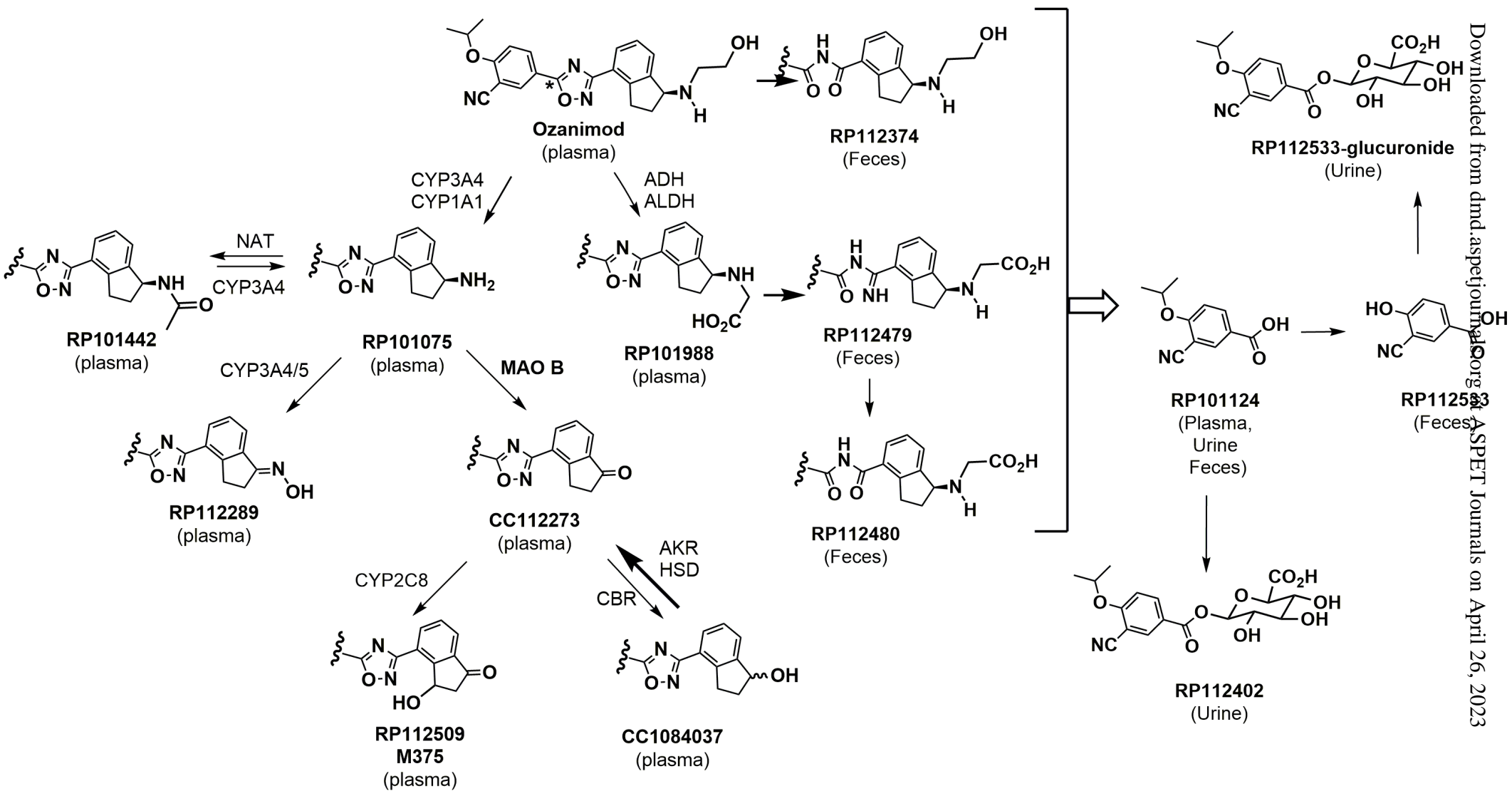


Figure 4
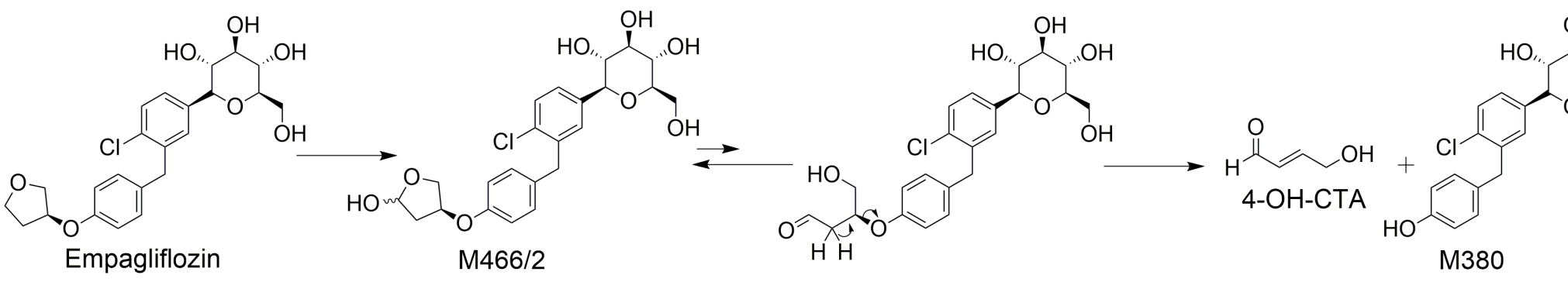


\section{Figure 5}

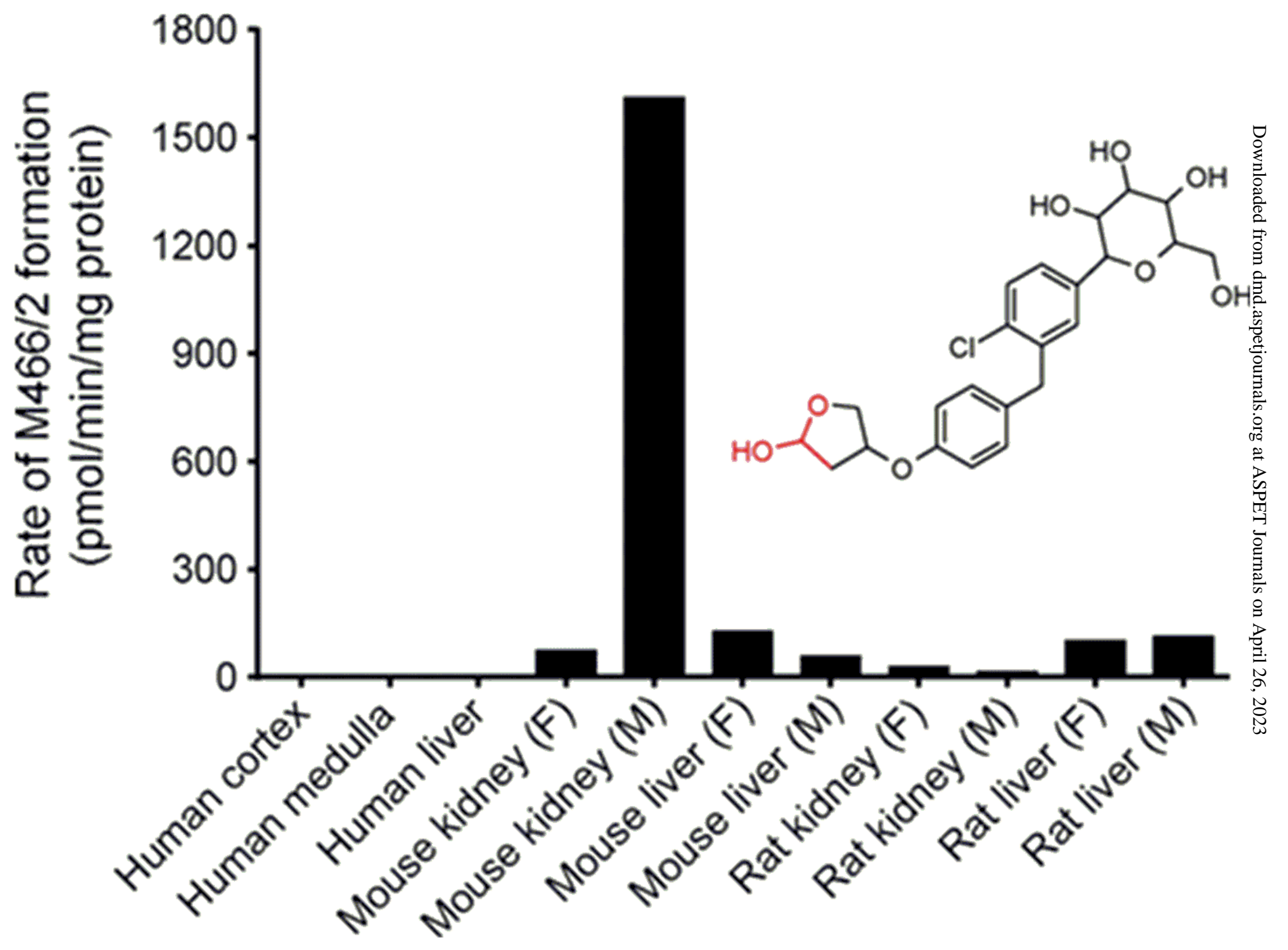




\section{Figure 6}
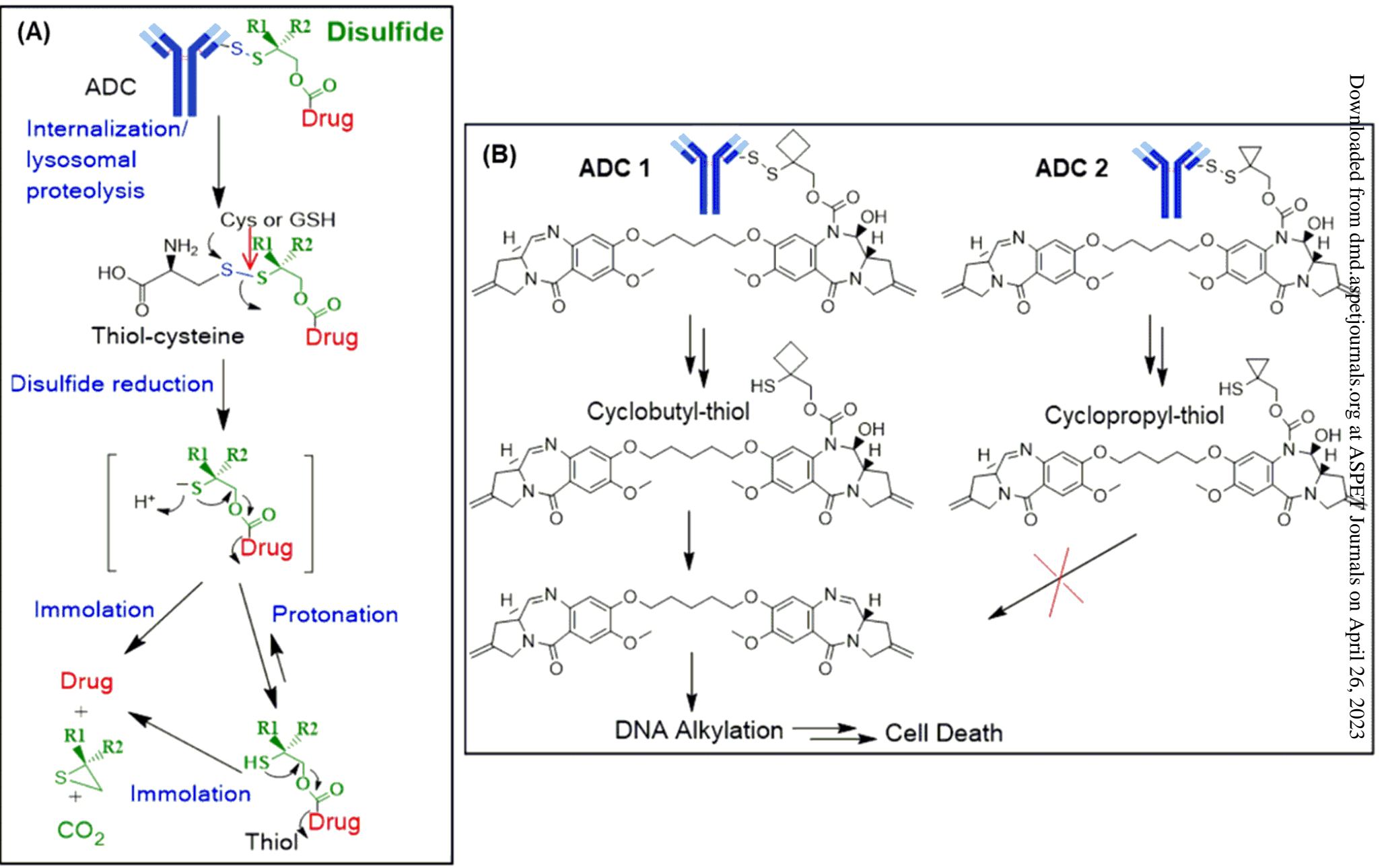